(1)

CrossMark

\title{
Novel coronavirus infection in newborn babies aged $<28$ days in China
}

\author{
To the Editor:
}

The outbreak of coronavirus disease 2019 (COVID-19) is spreading rapidly [1-8]. On 31 January 2020, the World Health Organization declared COVID-19 a public health emergency of international concern. By 13 March 2020, COVID-19 had been confirmed in 127 countries, with 145166 cases and 5428 deaths worldwide.

At an early stage of the epidemic, older adults were reported to be more likely to be infected [1-8]. Subsequently, infections in infants under 1 year of age were reported [8]. To date, little is known about the infection in newborn babies. Furthermore, data regarding intrauterine transmission are scarce [9]. In this study, we identified all infected newborn babies in China by 13 March 2020 and describe the clinical features, treatment, outcomes and intrauterine transmission potential.

For this retrospective study, we identified all neonatal infections with the COVID-19 virus, SARS-CoV-2, between 8 December 2019 and 13 March 2020 in China. The summary number of new infections was released daily by the National Health Commission in China [10]. We systematically explored all the 81026 cases that were laboratory-confirmed by 13 March 2020, as described previously [8]. In short, we retrieved the summary data from the central government and local health departments and screened for newborn babies < 28 days of age. Local hospitals, administrative offices and families of patients were interviewed through telephone or online communication tools. This study was approved by the institutional review board of Wuhan University School of Health Sciences. The need for informed consent was waived as part of the public health outbreak investigation.

Data on the demographics, disease onset, diagnosis, treatment and outcomes were collected using standard forms. To analyse the intrauterine transmission potential, data were collected on the mother's disease onset (symptoms, timing of symptoms onset relative to delivery), diagnosis, Wuhan linkage (living in or visited Wuhan, or directly contacting visitors from Wuhan), delivery (delivery methods, hospital level, protection level, gestational age at delivery) and mother-child contact (separation, breastfeeding).

To detect neonatal infection, nasopharyngeal swabs or anal swabs were collected during hospitalisation. Quantitative real-time PCR was used according to the recommended protocol [11]. IgM/IgG antibodies were not used in the present study because this technology has been developed recently and had not been widely used at this stage.

For the mothers of the newborn babies, computed tomography (CT) scanning was used for preliminary screening. Abnormal results included ground-glass opacity and bilateral patchy shadowing. Suspected infection was defined as abnormal results on CT scanning coupled with typical clinical symptoms, including fever, cough, headache, sore throat, shortness of breath and sputum production. Nasopharyngeal swabs were collected for detection of SARS-CoV-2 nucleic acid. Confirmation of infection in mothers was based on nucleic acid tests.

Based on the data sources we used in this retrospective study, four nucleic acid-confirmed neonatal infections were identified through systematic and comprehensive searching among the 81026 confirmed cases in China as of 13 March 2020 (table 1). All four patients were hospitalised. Three were male. The age at diagnosis ranged from $30 \mathrm{~h}$ to 17 days.

Two newborn babies had fever, one had shortness of breath, one had cough and one had no noticeable symptoms. The onset of disease occurred in hospital for two newborn babies and at home for two

@ERSpublications

Neonates are susceptible to SARS-CoV-2 infection. The symptoms in neonates were milder and outcomes were less severe compared to adults. Intrauterine vertical transmission is not impossible but direct evidence is still lacking. https://bit.ly/342jGK9

Cite this article as: Zhang Z-J, Yu X-J, Fu T, et al. Novel coronavirus infection in newborn babies aged $<28$ days in China. Eur Respir J 2020; 55: 2000697 [https://doi.org/10.1183/13993003.00697-2020]. 
TABLE 1 Characteristics of four hospitalised nucleic acid-confirmed infections in newborn babies

Patient 1

Patient 2

Patient 3

Patient 4

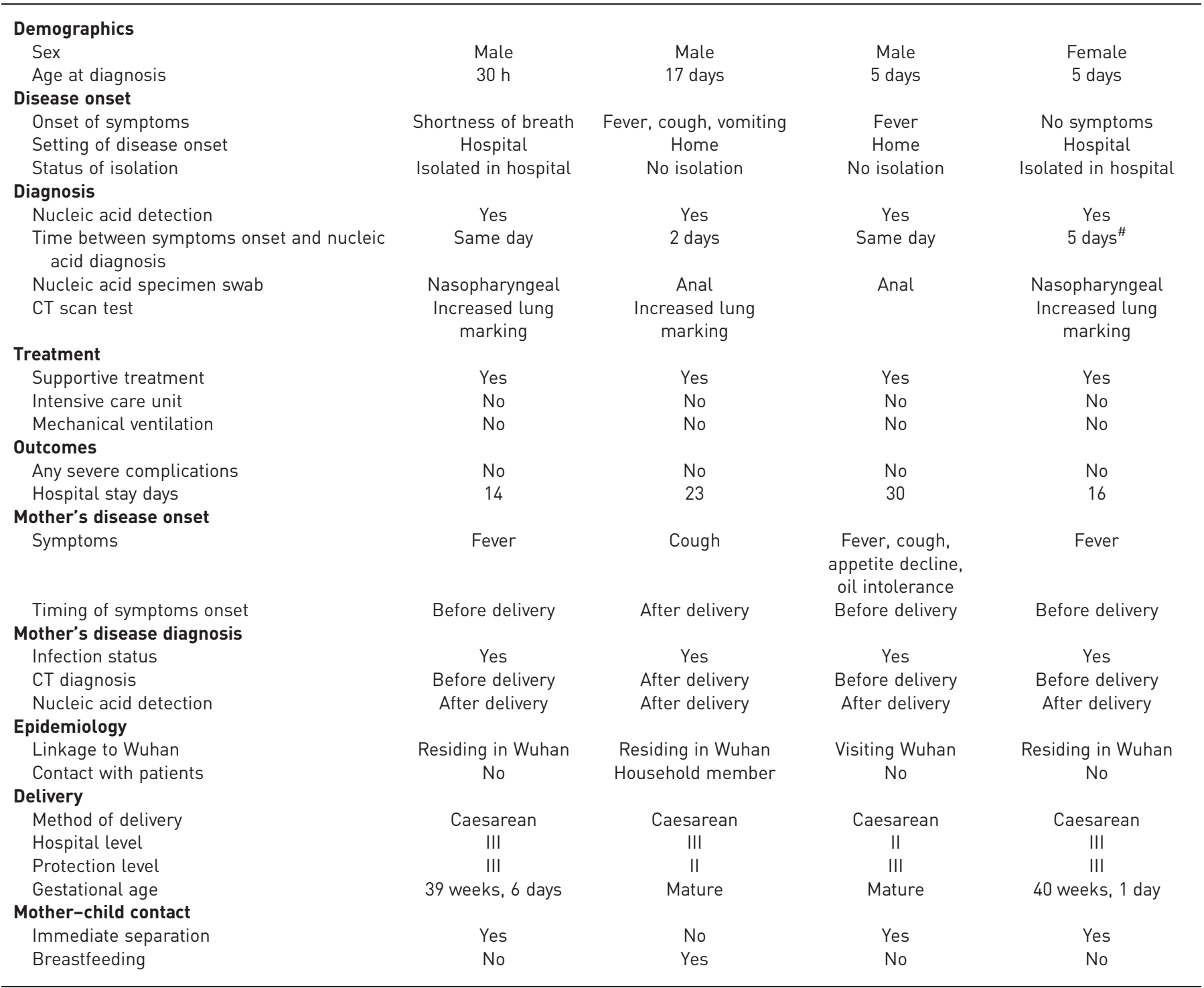

CT: computed tomography. ${ }^{\#}$ : time between date of birth and diagnosis.

newborn babies. Two newborn babies were in isolation and two were not in isolation at the time of disease onset.

Nucleic acid detection was performed using nasopharyngeal swabs for two newborn babies and anal swabs for two newborn babies. All four newborn babies tested positive for SARS-CoV-2 nucleic acid. CT scans were performed in three newborn babies. All showed increased lung marking. The time between dates of admission/symptoms and diagnosis was 0-2 days.

Supportive treatment was provided for all four newborn babies. None required intensive unit care or mechanical ventilation. None had any severe complications. Three newborn babies were deemed to recover after two consecutive negative nucleic acid tests (separated by $\geqslant 24 \mathrm{~h}$ ). Their hospital stay was 16,23 and 30 days, respectively.

All four newborn babies' mothers were diagnosed as infected. Three mothers showed symptoms before delivery and one after delivery. The most common symptoms in mothers were fever, followed by cough, appetite decline and oil intolerance. CT scans were performed for all four mothers, with three before and 
one after delivery. Abnormal findings were reported for all mothers. Nucleic acid detection was performed for all four mothers, with three after delivery and one before delivery. All four mothers tested positive for SARS-CoV-2 nucleic acid.

Caesarean section was used for all four mothers, three at level III hospitals and one at a level II hospital. The protection level was III for three mothers and II for one mother. Three newborn babies were separated from their mothers immediately after being born and were not breastfed; one neonate had not been separated from the mother and was breastfed for 16 days until symptom onset.

Based on these findings, symptoms of neonatal infection appear to be generally mild compared to adult patients. No severe clinical complications or deaths were observed in newborn babies, compared to fatality rates of $\sim 4-15 \%$ in adults [1-4]. Note that infants $<1$ year of age also presented mild or no symptoms and rarely severe complications [8]. On the one hand, it is encouraging that newborn babies and infants appear to have mild symptoms, although close monitoring for clinical deterioration is still necessary. On the other hand, mild or no symptoms in the youngest make it difficult to detect and prevent further transmission of this virus in the general population.

The number of neonatal infections was four, compared to the previously reported nine infections in infants aged between 28 days and 1 year [8]. Proportionately, the infection rate might be higher in newborn babies than infants. Different exposure chances may partly explain the different infection rates, but different transmission routes may be another explanation. Vertical transmission may be an additional route for infections in newborn babies but not possible for infants, although a previous study of six pregnant women did not find any direct evidence [9].

The findings of the present study suggest intrauterine transmission potential. All four newborn babies were delivered through caesarean section and three were under level III protection. The possibility of intrapartum mother-to-child transmission by vaginal delivery was excluded. Except for patient 2 having contact with an infected visitor, all the other three newborn babies were in isolation when symptoms occurred. No mother-child contact or breastfeeding occurred in these three newborn babies. The time between birth and diagnosis was limited, ranging between $30 \mathrm{~h}$ and 5 days. Taken together, the chance of infection through ways other than intrauterine transmission is deemed to be low. However, there are other explanations for neonatal infections. Firstly, the possibility of nosocomial infection cannot be completely ruled out. Secondly, nasopharyngeal and anal swabs cannot directly indicate intrauterine infection, and no viral particles were found in the amniotic fluid or cord blood from six patients [9]. Thirdly, the number of neonatal infections is small, although it is possible that the identification of cases was incomplete due to a lack of identifying information for newborn babies. Fourthly, SARS-CoV, another coronavirus with a similar genome sequence [12], was not found to be vertically transmitted [13, 14]. Further research is warranted.

There are limitations to the present study. First, although a systematic and comprehensive search was made for SARS-CoV-2 infection in newborn babies $<28$ days of age, incomplete identification of cases is possible. Secondly, the present study identified mostly symptomatic patients. However, asymptomatic infection exists for COVID-19 $[8,15]$. Thirdly, intrauterine tissue samples were not collected for these four newborn babies. Direct intrauterine detection of SARS-CoV-2 was not possible in the present study.

In summary, newborn babies are susceptible to SARS-CoV-2 infection. The symptoms in newborn babies were milder and outcomes were less severe compared to adult patients. Intrauterine vertical transmission is not impossible, and further studies are warranted to search for virological evidence.

Zhi-Jiang Zhang ${ }^{1,5}$, Xue-Jie Yu ${ }^{1,5}$, Tao Fu $\odot^{2,5}$, Yu Liu ${ }^{3}$, Yan Jiang ${ }^{2}$, Bing Xiang Yang $\odot^{4}$ and Yongyi Bi ${ }^{1}$

${ }^{1}$ Dept of Preventive Medicine, School of Health Sciences, Wuhan University, Wuhan, China. ${ }^{2}$ Renmin Hospital of Wuhan University, Wuhan, China. ${ }^{3}$ Dept of Statistics, School of Management, Wuhan Institute of Technology, Wuhan, China. ${ }^{4}$ Dept of Nursing, School of Health Sciences, Wuhan University, Wuhan, China. ${ }^{5}$ Contributed equally.

Correspondence: Zhi-Jiang Zhang, Dept of Preventive Medicine, School of Health Sciences, Wuhan University, No. 185 Donghu Road, Wuhan 430071, China. E-mail: zhang22968@163.com

Received: 1 March 2020 | Accepted after revision: 26 March 2020

Author contributions: Zhi-Jiang Zhang conceived the study and drafted the manuscript; Zhi-Jiang Zhang collected data; Xue-Jie Yu, Tao Fu, Yu Liu, Yan Jiang, Bing Xiang Yang and Yongyi Bi edited the manuscript.

Conflict of interest: None declared.

Support statement: This work was supported by the Fundamental Research Funds for the Central Universities (2042020kfxg04). 


\section{References}

1 Huang C, Wang Y, Li X, et al. Clinical features of patients infected with 2019 novel coronavirus in Wuhan, China. Lancet 2020; 395: 497-506.

2 Chen N, Zhou M, Dong X, et al. Epidemiological and clinical characteristics of 99 cases of 2019 novel coronavirus pneumonia in Wuhan, China: a descriptive study. Lancet 2020; 395: 507-513.

3 Wang $\mathrm{D}, \mathrm{Hu} \mathrm{B}, \mathrm{Hu} \mathrm{C}$, et al. Clinical characteristics of 138 hospitalized patients with 2019 novel coronavirus-infected pneumonia in Wuhan, China. JAMA 2020; 323: 1061-1069.

4 Yang $\mathrm{X}, \mathrm{Yu} \mathrm{Y}, \mathrm{Xu}$ J, et al. Clinical course and outcomes of critically ill patients with SARS-CoV-2 pneumonia in Wuhan, China: a single-centered, retrospective, observational study. Lancet Respir Med 2020; 8: 475-481.

5 Li Q, Guan X, Wu P, et al. Early transmission dynamics in Wuhan, China, of novel coronavirus-infected pneumonia. N Engl J Med 2020; 382: 1199-1207.

6 Liang WH, Guan WJ, Li CC, et al. Clinical characteristics and outcomes of hospitalised patients with COVID-19 treated in Hubei (epicentre) and outside Hubei (non-epicentre): a nationwide analysis of China. Eur Respir J 2020; 55: 2000562.

7 Guan WJ, Ni ZY, Hu Y, et al. Clinical characteristics of coronavirus disease 2019 in China. N Engl J Med 2020; 382: $1708-1720$.

8 Wei M, Yuan J, Liu Y, et al. Novel coronavirus infection in hospitalized infants under 1 year of age in China. JAMA 2020; 323: 1313-1314.

9 Chen $\mathrm{H}$, Guo J, Wang $\mathrm{C}$, et al. Clinical characteristics and intrauterine vertical transmission potential of COVID-19 infection in nine pregnant women: a retrospective review of medical records. Lancet 2020; 395: 809-815.

10 National Health Commission of the People's Republic of China. www.nhc.gov.cn Date last accessed: 14 March 2020.

11 World Health Organization. Coronavirus disease (COVID-19) technical guidance: laboratory testing for 2019-nCoV in humans. www.who.int/emergencies/diseases/novel-coronavirus-2019/technical-guidance/laboratory-guidance Date last accessed: 10 February 2020.

12 Zhu N, Zhang D, Wang W, et al. A novel coronavirus from patients with pneumonia in China, 2019. N Engl J Med 2020; 382: 727-733.

13 Shek CC, Ng PC, Fung GP, et al. Infants born to mothers with severe acute respiratory syndrome. Pediatrics 2003; 112: e254.

14 Wong SF, Chow KM, Leung TN, et al. Pregnancy and perinatal outcomes of women with severe acute respiratory syndrome. Am J Obstet Gynecol 2004; 191: 292-297.

15 Chan JF, Yuan S, Kok KH, et al. A familial cluster of pneumonia associated with the 2019 novel coronavirus indicating person-to-person transmission: a study of a family cluster. Lancet 2020; 395: 514-523. 